\title{
Virtual Clothing Display Platform Based on CLO3D and Evaluation of Fit ${ }^{\star}$
}

\author{
Yan-Xue Wang, Zheng-Dong Liu* \\ No. A2, East Yinghua Street, Chaoyang District, Beijing 100029, China
}

\begin{abstract}
The article uses CLO3D and HTML5 to design a virtual clothing display system. And for the problems that may arise when using CLO3D to make garments, the solution is given. At the same time, the evaluation of the fit of the virtual clothing made in CLO3D was discussed. Designers can use CLO3D to quickly create virtual clothing in accordance with the users' requirements. After, through the system, designers can publish it on the Web for the users to effectively see the process of cloth making in an all-rounded way, while sustaining communication with designers. The design of this virtual clothing display platform provides some reference value for the digital model of the apparel industry.
\end{abstract}

Keywords: 3D Clothing Design; CLO3D; Virtual Display; Virtual Fitting

\section{Introduction}

With the development of society and the advancement of technology, all walks of life have more or less contact with the Internet. Clothing, as the "second skin" of the human body, In addition to basic functions such as prevention and warmth, it is a label that shows one's own personality and reflects personal style. People pursue individualized and diversified clothing styles. Therefore, people are no longer just satisfied with traditional and mass-produced clothing styles, but hope to have a clothes that belong to their own independent labels. Thus, a growing number of niche and independent designers has sprung up. They are not mass-producers like traditional large clothing companies are. Instead, they will design a niche and very fashionable clothes based on their own design style and customer needs. Because of the personal characteristics such clothing represents, it is usually not suitable for large volume production. With regard to both, the number of production and production cost, it is very difficult for personal brand designers to use the Internet, television and posters for large-scale publicity or to have models present their work. Therefore, starting a personal brand website, establishing the human body model by creating a virtual sample of it and publishing it to a virtual display system, would shorten the production

${ }^{\star}$ Project supported by the Beijing science and technology program (No. Z171100005017004).

${ }^{*}$ Corresponding author.

Email address: jsjlzd@bift.edu.cn (Zheng-Dong Liu). 
cycle and the production costs as well as it would facilitate the communication between designers and users. This would help designers to design clothes that are more in line with consumer needs.

The site is designed with CLO3D and HTML5. The project consists of two major components: the clothing design part of CLO3D and the website implementation part of HTML+CSS+JavaScript. And in the CLO3D, the fit of the clothing has been evaluated.

\subsection{CLO3D}

This is a software that fashion designers use to complete garment design and production. It can completely meet a series of design requirements that fashion designers might have, such as fashion design, pattern making, adjustment and modification, fabric selection, display and release. For designers, this is a very convenient software. It can quickly implement the designer's design prototype. Using this software can not only greatly reduce the length of the design process, but also save on the cost and time of production, making it a very convenient and practical clothing design software. At the same time, we can also make a certain evaluation of the designed virtual clothing through this software. In this way, ensure that the designed clothing is more in line with the needs of human comfort and fit. (Statement: all of the pictures in this article, namely, mannequin is CLO3D publicly model and clothing pictures are for my own production and do not involve any copyright issues.)

\subsection{Project Significance}

Regarding the way clothes are displayed, there are a few other ways besides television, the Internet, posters of plane publicity and model show. The above-mentioned displays either require having a physical object to promote, or the display is not entirely $3 \mathrm{D}$, thus not offering comprehensive clothing details. This not only requires a lot of time to make a sample of a dress, but also needs to consider the extra promotional costs, which are paid to other platforms that provide advertisement services. For some personal brand designers, because they produce a small amount of clothing items, making use of the publicity does not sound very reasonable. Therefore, the simple virtual clothing display system formed by CLO3D and HTML5 can provide with a very good solution to the problem: As the clothes are designed virtually, one can not only see the effect of wearing and style but also details such as fabrics is visible. This can be published using the HTML website. Publishing it on the Web makes it easier for the consumers to have a better image and understanding of what the clothes look like and at the same time it gets rid of the dependency on the professional production software.

\section{Research Status at Home and Abroad}

At present, for virtual clothing display and virtual fitting, firstly, the data indicators of the human body are usually detected by 3D anthropometric technology. Then, by digitally fitting and digitizing the pieces on the mannequin, the pieces are stitched together to form a virtual 3D clothing display system. For garment production, generally, a rough board of clothes is made through 2D page at first or by clothing CAD to obtain precise clothing plate with corresponding edge stitch and by adjusting the related parameters and angles and keep the relevant information. 
Then, by performing pressure point matching and stitching on the network point of the 3D mannequin, a 3D stereo effect is formed, realizing thus an effect of displaying the virtual clothing [4]. My Virtural Model is a relatively mature virtual costume presentation website. Customers can select their favorite avatars and models with similar body shapes, or they can upload their own avatars to achieve more authentic effects. By choosing different clothes and models, the model also changes since the system can analyze and compare the fit degree according to the size of human body and the size of the clothing clipping. This allows it to recommend the most suitable style and size for customers. Some laboratories have developed more mature virtual garment fitting systems such as MIRCloth system developed by MIRAlab in Geneva, Switzerland, C-Me and V-Stitcher of Browzwear company in the United States [1].

However, the current big problem with the display of virtual clothing is that some fabrics cannot be displayed very realistically. This remains a crucial problem in the display of virtual clothing together with the actual facial and body characteristics for consumers. Both are areas that need improvement.

\section{The Overall Design}

The operation flow chart is shown in Fig. 1. First of all, through the CLO3D software, making virtual clothes designed by designers. And evaluate the fit and comfort of the clothing. Modify and adjust the clothing according to the evaluation situation. Then post the adjusted clothing on the web. It will be included style diagrams, detail diagrams, and dynamic videos. Consumers can see all relevant information about clothing on this page. When they are dissatisfied with the style of clothing, they can communicate directly with designers online to make timely, efficient and accurate changes to clothing.

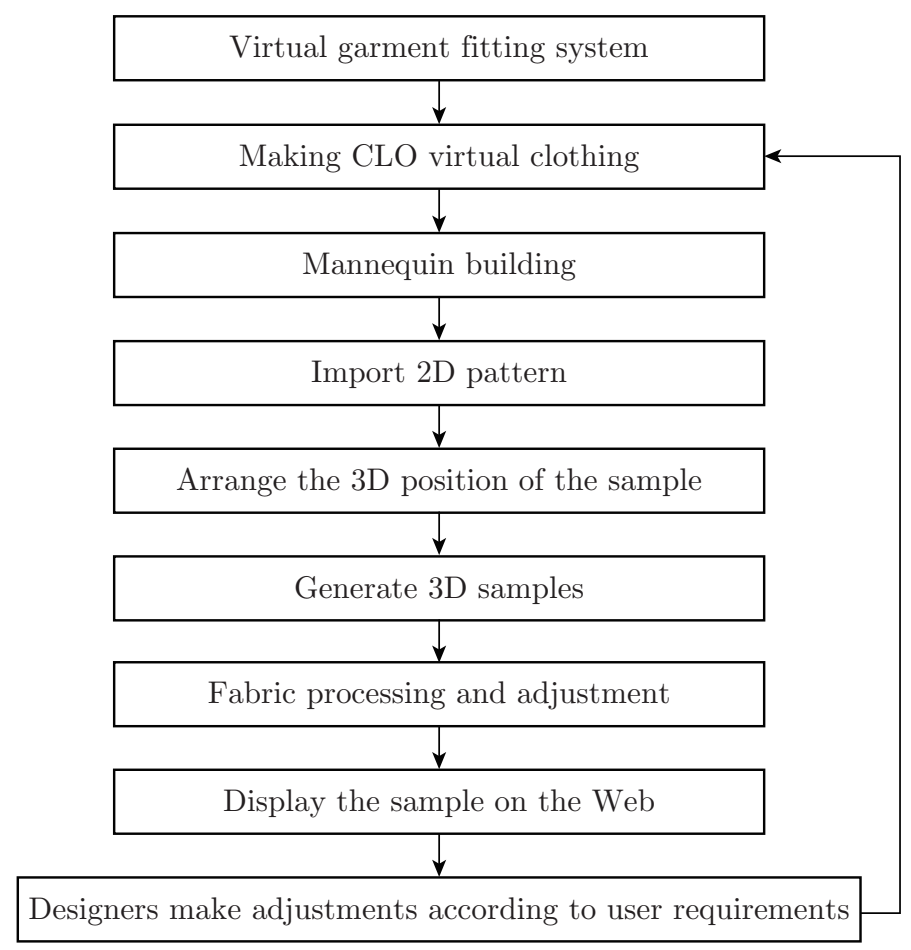

Fig. 1: Virtual Display System Process Diagram [7] 


\section{Concerns in Virtual Design}

\subsection{Establishment of Mannequin}

When making and designing virtual clothing, the first step is to choose a mannequin as the basis. This is an important basis for garment modification and display on 3D platform. Therefore, the size, shape and posture of the models play a very important role in 3D, as shown in Fig. 2 below. In the display, the model is divided into two kinds of display effects: dynamic and static. In the static display, the model is equivalent to when the person is standing still and at this time, it is convenient to observe the drape effect of the garment at rest. The dynamic display is the effect when the model is exercising and this is equivalent to the effect of people walking normally. It is generally convenient to observe the overall effect of the clothing. The dynamic display of the general effect is still in the model, adding to its specific movements and gestures it can be dynamically displayed. Therefore, before designing a garment, the design of the mannequin is particularly important. Designers need to adjust the parameters such as height, weight, chest circumference, waist circumference, hip circumference, head circumference etc., as much as possible to make sure the design is as close to the user's actual body shape as possible $[2]$.

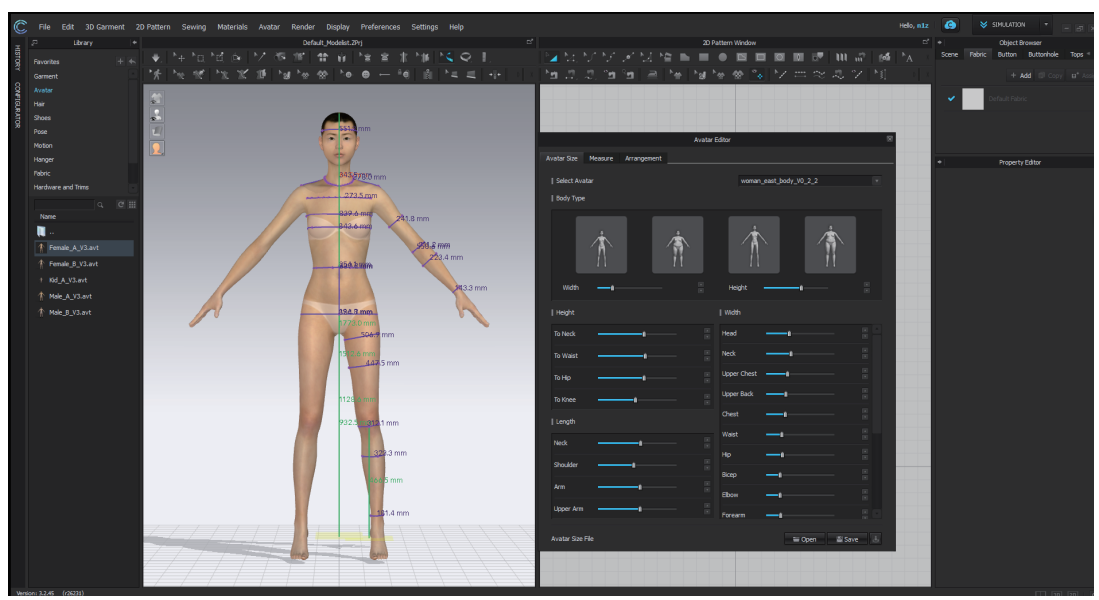

Fig. 2: Mannequin Building [7]

\subsection{Arrange the Arrangement of Points}

If you want to design clothes on $3 \mathrm{D}$ models, the first step is to create a $2 \mathrm{D}$ version on a $2 \mathrm{D}$ layout. This part also can use CAD import directly: after the 2D plate suture, the 3D layout automatically synchronizes with the 2D layout; then click the arrange point on the 3D page. This step is very important. As shown in Fig. 3, when you are ready to place the arrangement points, you need to organize the layout around and make sure that these points are positioned in the right place and are as close as possible to the model, in order to avoid any disorder during fitting and also to make it convenient to suture. In the process of suturing, the suture tool should be selected to stitch the corresponding position of the suture together. When there are a lot cloth pieces, it is necessary to suture from the top to the bottom and then from the inside to the outside. At this point, the 3D interface will be stitched together synchronously. By clicking "simulation", the 
clothes will be worn on the model according to the previous arrangement and design. In the end, you can finally check it and adjust it.

\subsection{Fabric Adjustment and Matching}

After the completion of sewing, the fabric attributes need to be selected. For some special patterns, it is often necessary to edit and process the required patterns by using Photoshop. After achieving the desired effect, import them into the CLO3D to adjust the corresponding clothing to achieve the overall effect. If you are not satisfied with the imported fabric and you want to adjust it to an optimal state, you can modify relevant parameters, such as color, brightness, highlights, tightness, fabric direction, etc.. This is shown in Fig. 4 [5].
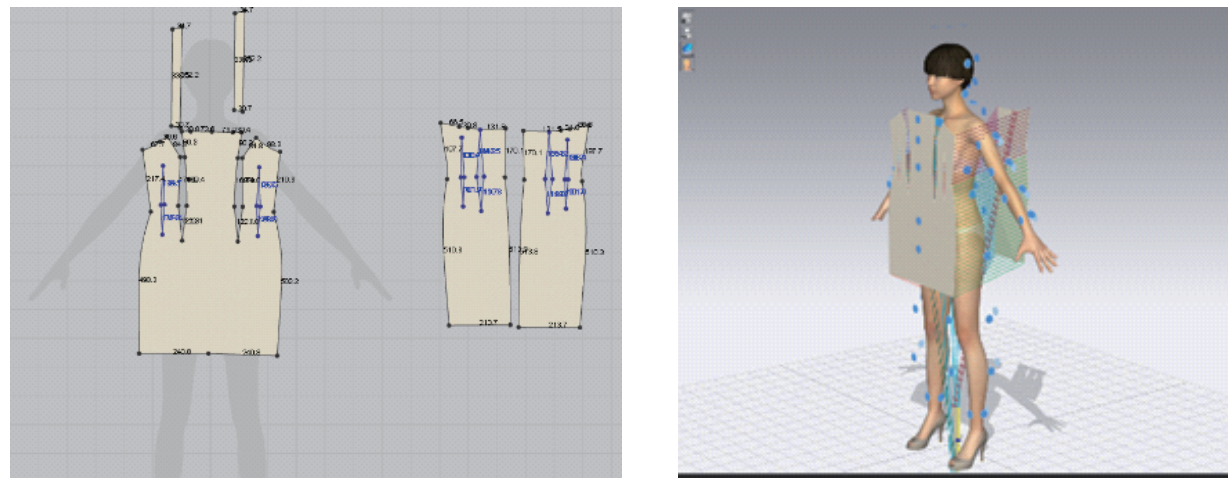

Fig. 3: Sewing Clothes and Place Arrangement [7]

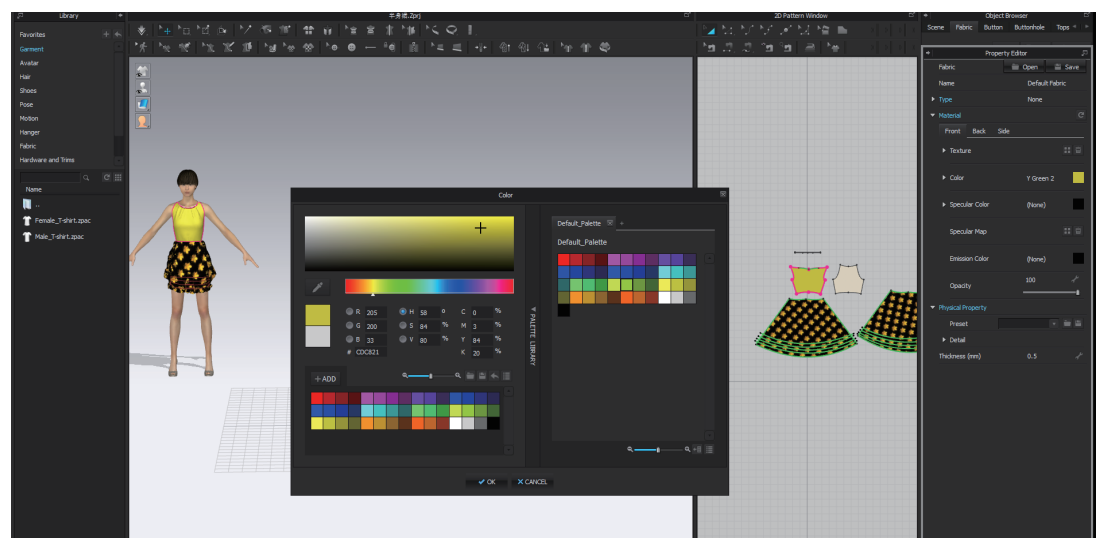

Fig. 4: Fabric Design [7]

\subsection{The Overall Comfort Test and the Fit Assessment}

By using CLO3D you can have a comfortable judgment on the finished clothes, and the range of pressure distribution and the distribution of contact points contributes to the judgment. The distribution of pressure can reflect the stress level of the clothes when they are worn on the model. We can distinguish according to the color of the display. If the cloth is being stretched really hard, it is shown in red, and for the opposite it is shown in the green color. The red area is 
larger, meaning that the clothing has a more oppressive feeling. However, if the area of green is bigger, the clothes have less pressure on the human body. Contact point is used to observe closely the extent of contact between the human body and clothes. If the contact points are distributed very evenly, it means that the clothes do not cause a sense of restraint to the person. This is demonstrated in Fig. 5 [2].
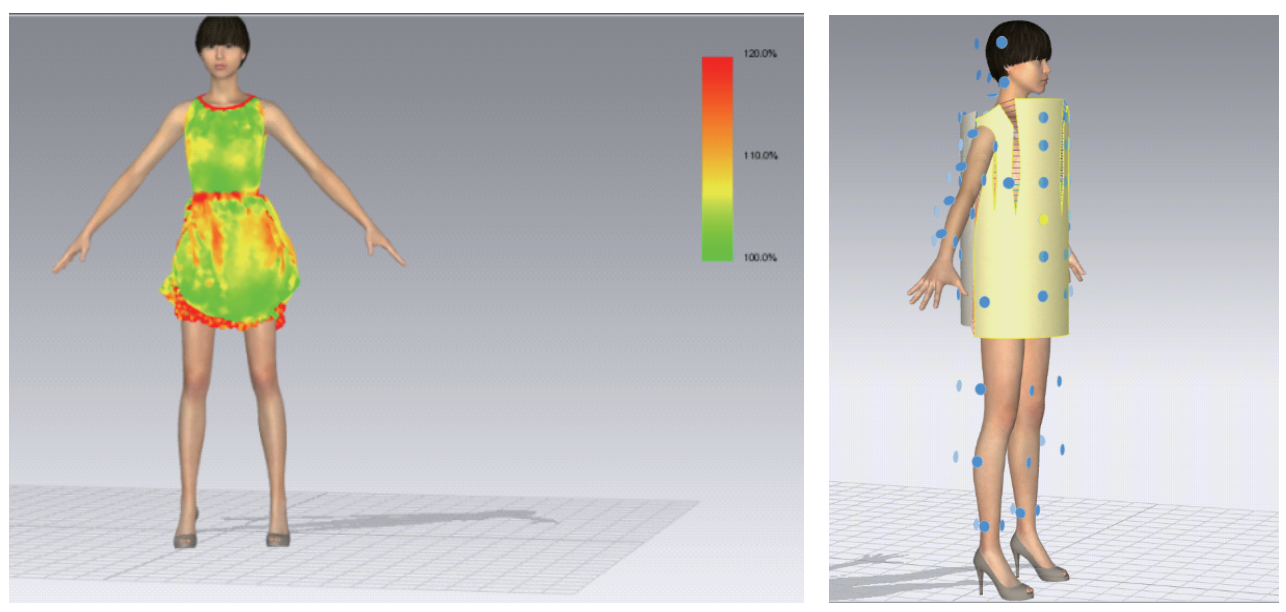

Fig. 5: Pressure Profile [7]

Through the distribution of the pressure of the clothing, it can be very intuitive to see the pressure of the virtual model when wearing the clothing, the unit is "kpa". In addition, we can also judge the suited clothes according to some other values.

(1) Fit map

The fit map is evaluated for whether the virtual model is suitable for wearing this dress. This is shown in Fig. 6, It can be seen that the clothing on the model is divided into two colors. Yellow stands for a specific position that feels tight, and the proportion of the total clothing; Red represents the area where it can't be worn and the proportion of the overall clothing. In the yellow area, you can adjust according to the effect achieved by the clothes. However, the red part must be adjusted, otherwise the red part of the actual clothing cannot be worn normally.
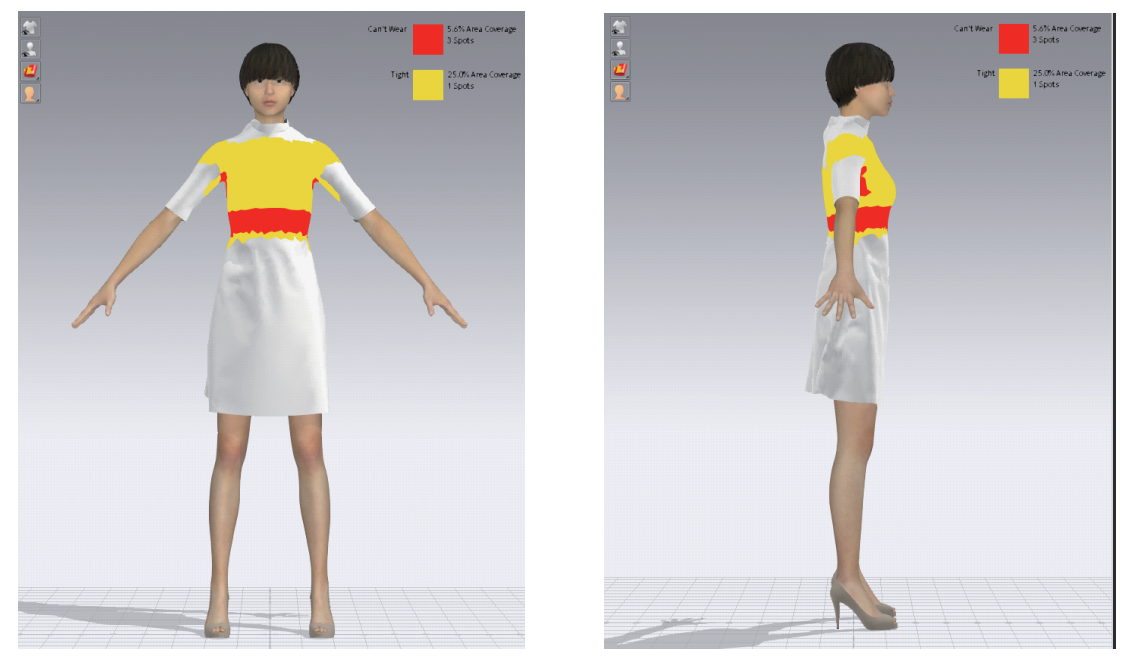

Fig. 6: Fit map 
(2) Transparent surface

The transparent surface shows the state of the garment structure on the virtual model in a transparent state when all the fabrics and patterns of the garment are removed. Designers can visually see the gaps and fit between the garment and the virtual model through transparent surface. This is shown in Fig. 7, through the display of transparent surface, designers can judge the overall fit and looseness of the garment based on experience and modify it. In the fit map, it is shown as unwearable.
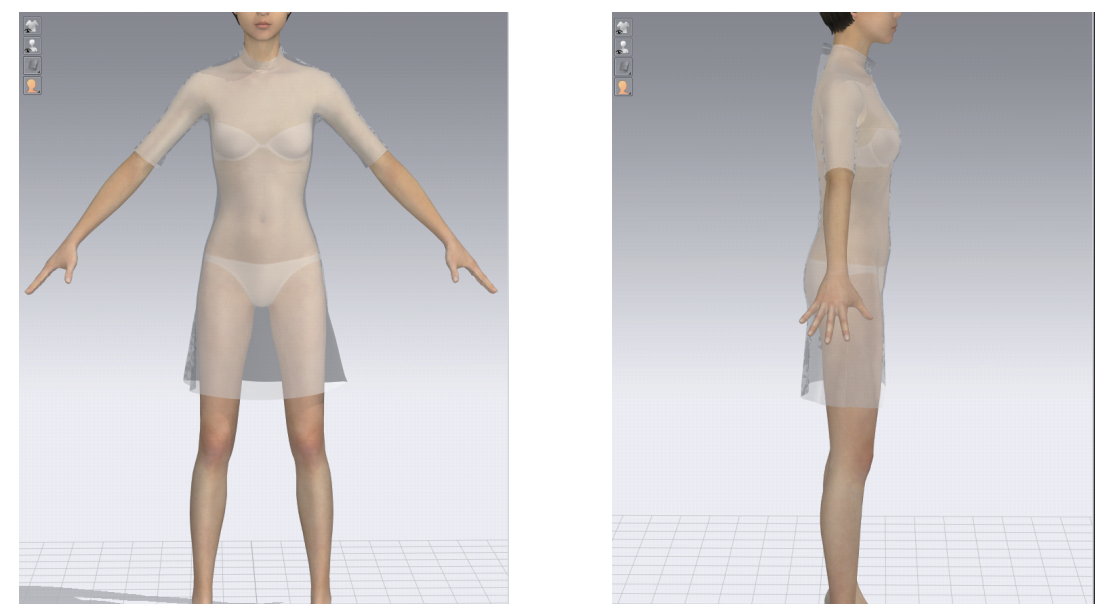

Fig. 7: Transparent surface

(3) Mesh pattern

This is shown in Fig. 8, through the mesh pattern, you can see the mesh density of the clothes. In the fabric of the garment, the spacing between particles is smaller, the mesh is denser, the structure of the fabric is finer, the simulated effect is better. But too much time can also cause a lot of work. Therefore, we should try our best to make choices. While pursuing fabric materials, also predict the workload of document processing. If the process is too time-consuming, it will not only waste a lot of time for the designer, but also add a lot of work when synchronizing to the webpage.
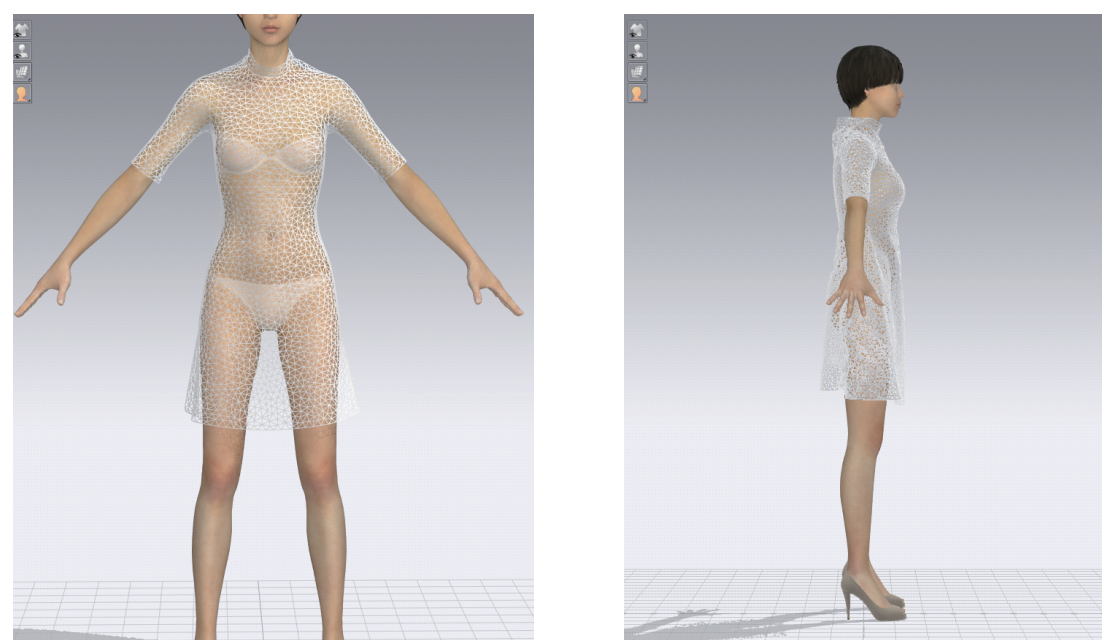

Fig. 8: Mesh pattern 
(4) Strain map

The strain map and the pressure map are very similar in the intuitive look. The difference is that the pressure map reflects the pressure of the garment on the virtual model, and the strain map indicates the degree of tension caused by the pressure itself. This is shown in Fig. 9. Through the strain map, it can be seen how much the deformation of the garment fabric occurs after being worn on the virtual model. If the area shown by the fabric is red, the fabric is stretched more than $120 \%$. At this time, corresponding to the previous introduction, clothing pressure on the human body has more than the human body can bear. In the fit map, it is shown as unwearable; the orange to yellow area indicates that the stretch of the fabric is between $110 \%$ and $120 \%$. This means that although the human body has a sense of urgency when wearing it, it is affordable. For some tight-fitting clothes, it can be within this tolerance; Green indicates that the fabric is in normal condition. That is, the human body does not stretch the fabric. At this time, the person wearing this dress feels loose and not bound.
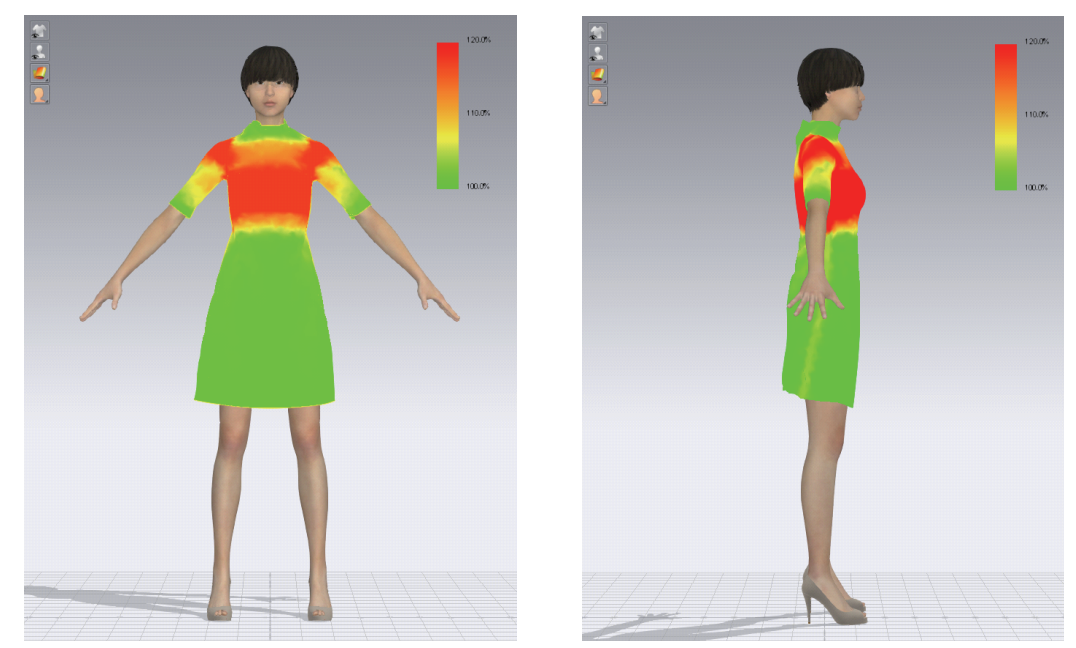

Fig. 9: Strain map

Since the clothing is virtually manufactured, there is no actual scenario to experiment with it. Therefore, the designer can adjust the already formed garments and improve them by the methods shown in the above several figures. In general, the structure and size are already made according to standard data, so there is not much room for modification. Then the adjustments should be modified on the parameters of the fabric itself. This is shown in Fig. 10. This picture is the modification interface of the fabric parameters. When the fabric is too tight, the shrinkage weft is generally adjusted. The elasticity of the overall weft expansion and contraction of the fabric change. The shrinkage weft is larger, the elasticity of the latitude is greater. When the clothing and body are too obedient, designer needs to adjust the fabric pressure to make the fabric stiffer. The pressure is greater on the fabric, the fabric is stiffer, and the obedient degree is smaller between the body and the human body. Generally, the fluffy fabric or down jacket is made by changing the fabric pressure to change the shape of the fabric, thereby simulating the overall effect. Although these methods cannot give some specific and accurate values like some professional equipment, conduct a quantitative assessment of comfort and fit. But for the design of virtual clothing, there is a great reference value, which can give designers and consumers a qualitative feeling. 


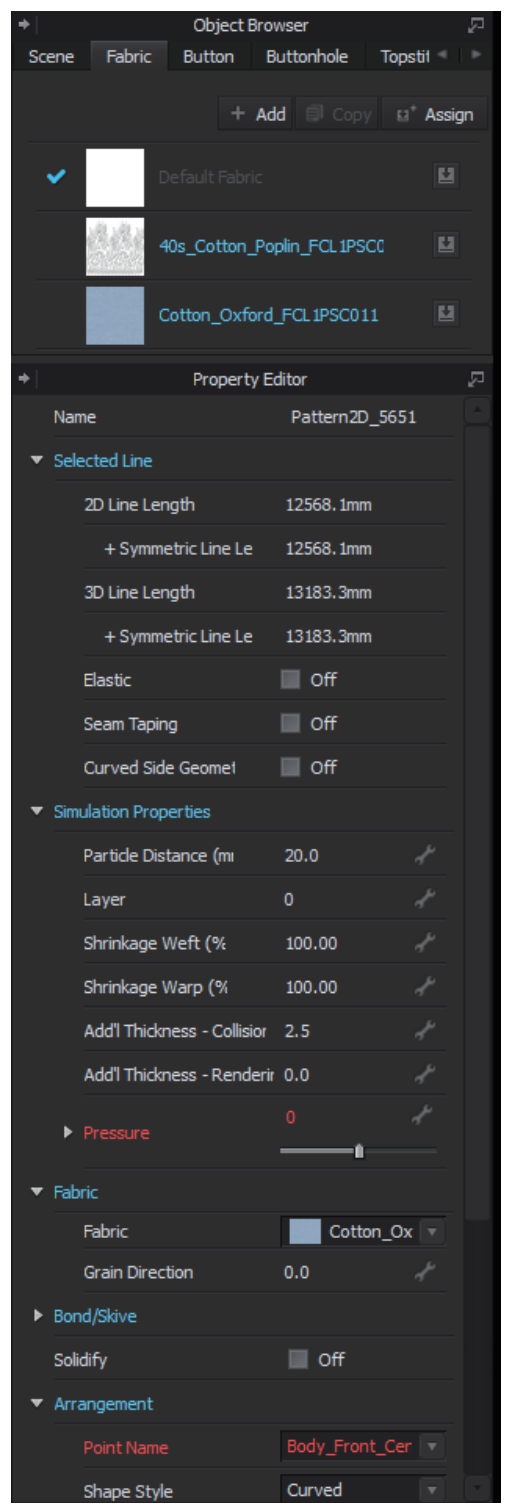

Fig. 10: Property editor

\section{The Web System Analysis and Design}

\subsection{Requirements Analysis Background}

With the development of technology and the Internet popularity, the latter is affecting the way people use Internet to work, live and study. People are used to getting information through Internet explorer, thus no matter the industry, it will be inevitably linked to the Internet.

The clothing-related businesses, although they are more traditional, Internet still has an impact on them. In the previous designs, designers need to carry out a large number of tedious patterning and modification processes. This would mean that not only does the process cost a lot of time, money and material resources, but it also means that users cannot directly see the style they want. Only once the product is completed, they can see whether or not it meets their expectations. If they find that the product did not meet their expectations, another change is inevitable. This 
repeated, tedious and wasteful mode of operation will not promote the garment industry toward a more perfect direction and the emergence of the Internet seems to break this bottleneck.

The development of additional software that provide making and design pattern has solved some of the problems of manual pattern. Using the software to dress, not only can be simple and accurate, but it is also more convenient to modify and conduct mass production. This means that the designer's work efficiency and creative inspiration can be greatly improved. For users, e-commerce as a convenient and fast transaction mode can also be a platform for designers to show their product orders to ordinary users. When designers are not able to show their finished graphics on professional software to users, they can upload them to relevant webpages through HTML5 publishing and users can more easily and intuitively check for the style of clothes they need. These series of operations are carried out through the Internet, they significantly save time and money and add to the friendly experience between designers and users.

The design of the front-end display system is mainly used to connect the designer with the user through a web page. The user can see the details of the clothes and the dynamic display of models on the web page as well as leave a message below and communicate with the designer directly to modify the clothes.

\subsection{Overall Page Design}

The overall design of the web page is relatively simple and clear. The overall page is shown in Fig. 6, with CSS+DIV for the overall layout. CSS+DIV is a method of web page layout and unlike the usual table layout positioning, the CSS+DIV can achieve the separation of the outer outline and content of the page. This paper adopts the embedded system.

Firstly, use DIV to divide the page into four sections, and divide the types of clothes. This is shown in Fig. 11 [3].

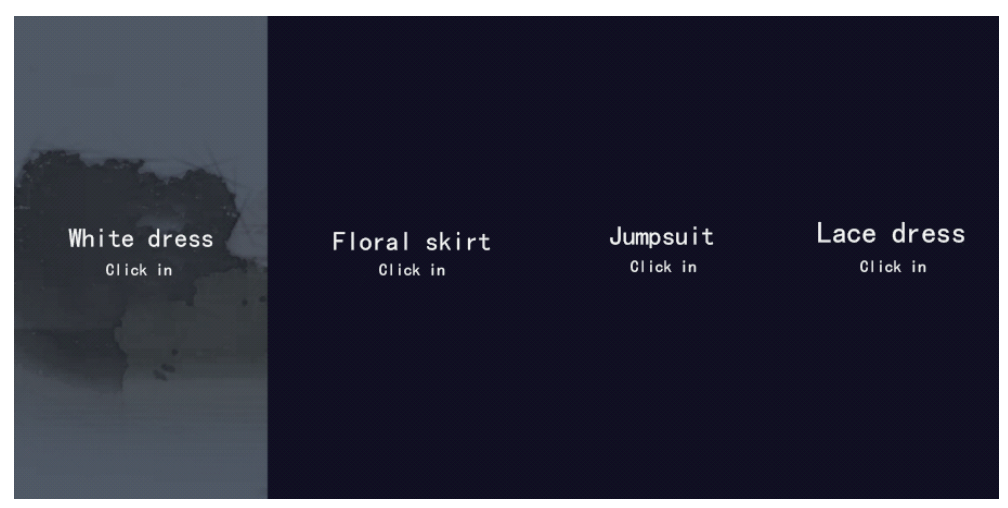

Fig. 11: Featured Listing [7]

Secondly, the reasonable layout of the page is very important in the design. Generally it is divided into the perception of the viewport, the adaptation of the responsive image, and the switching of the resolution. For viewports, there are generally three types. This article uses an ideal viewport, which is automatically matched and adjusted during web terminal display.

For the adaptation of responsive images, this paper chooses resolution switching. That is reserved under different scenarios as well as the ratio aspect of the picture content, allowing for the picture resolution to be changed. 
Finally, after entering the selected clothing page, you will enter the product details page, as shown in Fig. 12. In this page, you can see all of messages about the cloth. Including the dynamic video, static display, detail display, composition of fabric, creation ideas and other relevant information on the clothes. You can also know about a virtual model based on your body feels when wearing this outfit. The right part of the page is the designer's introduction, and below is the channel for communication with the designer, which can be used to communicate with the designer personally. If you have any dissatisfaction or other ideas, you can communicate with the designer in time. The designer will modify it as soon as possible according to your needs [3].

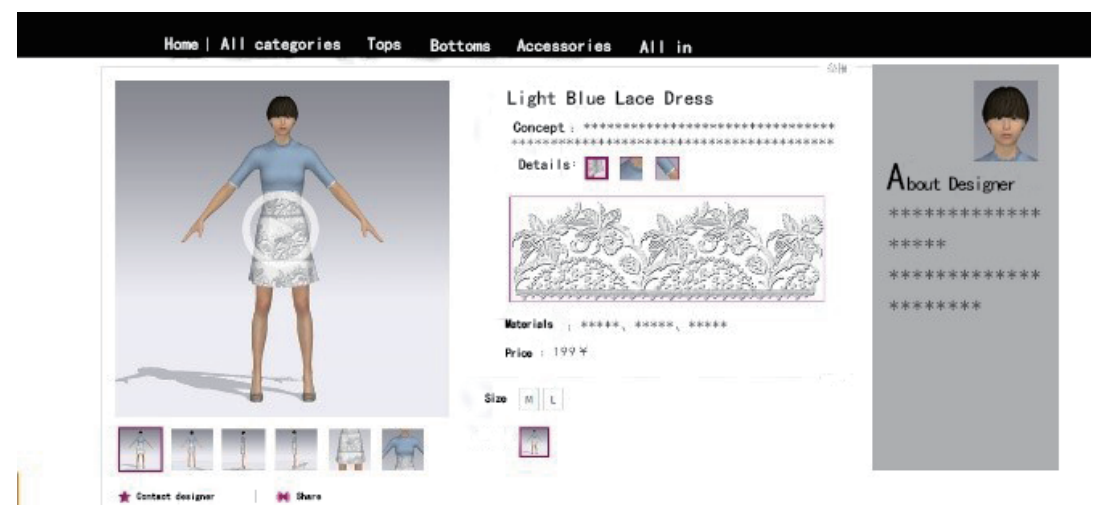

Fig. 12: Detailed Page Display [7]

\section{Conclusion}

The survey on domestic and foreign virtual clothing display platforms found that there indeed exists a big gap in this area, which means that there is still a great potential for development and room for improvement. Some online clothing e-commerce platforms basically use pictures to exhibit their clothes. The 3D virtual clothing display platform designed in this paper can serve as a good communication bridge between consumers and designers. Designers can easily express their design inspirations by making a set of virtual clothing. They do not need to spend a lot of energy and money to afford them. This paper also gives solutions to some problems that need to be paid attention to in the production process. After the completion of the virtual garment production, the comfort and fit of the garment were analyzed. The purpose is to make the virtual clothing can be more realistic to restore the real clothing brought to the user's feelings. It can be presented to consumers in a virtual way. Its effect is very close to reality and the user's ideas and opinions on clothes can directly be communicated to the designer, so that the designer can improve the clothes based on the feedback.

This article combines CLO3D with HTML5 to develop a virtual clothing display system for niche designers and customers. The biggest characteristic of this system is that: On the one hand, for designers, after users put forward the requirements of clothes, it is very convenient and fast to complete a set of virtual clothing display without physical objects and at a very low cost. At the same time, the software can also perform a certain evaluation on the fit and comfort of the clothing, and provide a certain reference for customers. On the other hand, users can directly see the details of the clothes and their dynamic effect as well as are able to communicate with 
designers in time for any further modifications.

To note are the details of the adjustment on CLO3D. The system still has a lot of room for improvement in the later user communication and detail restoration.

\section{$7 \quad$ Future Work Prospects}

The following improvements can be made as a response to the problems and deficiencies in the whole design process:

(1) Regarding the aspect of clothing production, the details of clothes can be made more precise, and the styles of some clothes should be designed better to facilitate the choice of users.

(2) When making a video presentation, it is better to make a simple processing of the background and choose appropriate pictures based on the theme of clothes. In this way, the impact will become more intense and the user experience will become better.

(3) In the process of web page creation, the starting idea is that a distinction can be made from the homepage, and for a preference, the background is not set to the relevant clothes but other pictures. To really make use of this in the future, we can change the background to a picture of the relevant category, which will be clearer at a glance.

(4) In video production it is actually more cumbersome. First, after making a good video site, it generates a link to the relevant page of code that can be used under each page category only to put a video. The best improvement is that you can make a database out of a video for a list of clothes. The link below is a link to this database and if designers have a new look of clothing design they can simply sort it into the database according to the appearance of the clothes. In this way, you only need to update the database regularly, making the daily maintenance of the webpage easier and more convenient.

(5) If it is possible, this software should also add a function that users can leave messages, which can be directly seen by the designer who designed the dress. In this way, when one of the users and designers is not online, it is convenient for the designer to directly make modifications and adjustments according to the needs of users, thus saving time.

\section{Acknowledgement}

I want to show my appreciation and gratitude to my tutor, Zhengdong Liu who provided a lot of help and guidance. This project was supported by the Beijing science and technology program (No. Z171100005017004)

\section{References}

[1] Tian M, Li J. Design mode and development trend of intelligent clothing. Journal of textile industry: 2005; 07.

[2] Liu J. Digital system for human body surface information collection and analysis. Chinese patent: 03121468.1; 2003-3-28. 
[3] Xu L. HTML and CSS front page design. Beijing: China hydraulic and power press: 2006; 05.

[4] Yi LL. Intimate design of intelligent clothing and analysis of its development potential. Journal of textile: 2015; 07.

[5] Tang SG. Creativity + : Photoshop cs4 web design, color matching and special effects case essence. Beijing: tsinghua university press: 2010; 7 .

[6] Wang ZZ. JavaScript web effects examples. Beijing: tsinghua university press: 2006; 09.

[7] Wang YX, Liu ZD. Research on the Application of CLO3D in Virtual Sample. Testile Bioengineeing And Informatics Symposium Priceedings, 2019: 1942-3438. 